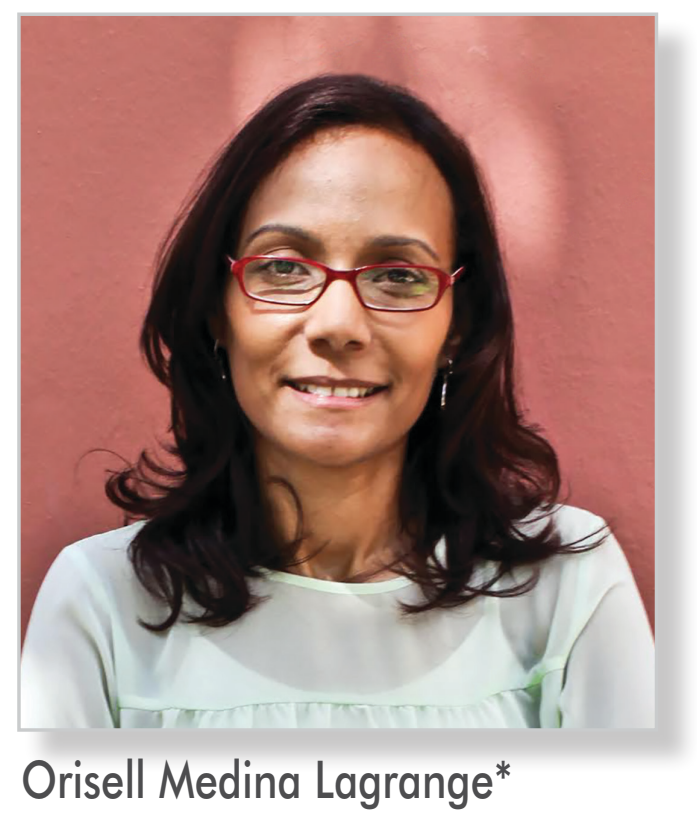

\title{
El proceso de producción escrita de la tesis de grado en Arquitectura: una vía para transformar a los estudiantes de pregrado en escritores académicos
}

\author{
The production process of the written \\ thesis in architecture: \\ a way to transform undergraduate students \\ into academic writers
}

Recibido: 10-06-14 Aprobado: 27-08-14

\section{Resumen}

Este artículo se deriva del proyecto de investigación-acción realizado durante el Diplomado de Lectura y Escritura a través del Currículo en el Nivel Superior, del Programa de Alfabetización Académica del Centro de Excelencia para la Investigación y Difusión de la Lectura y Escritura de la PUCMM. El objeto de estudio de esta investigación fue el proceso de enseñanza aprendizaje de la escritura en la asignatura Propuesta de Proyecto de Grado de Arquitectura. Los datos fueron recopilados en el período académico septiembre-diciembre de 2013, con el propósito de determinar las estrategias que facilitan la mejora de la escritura académica en los estudiantes de último año de Arquitectura. Las conclusiones principales fueron que al conocer el proceso de producción escrita y los elementos del discurso, los estudiantes se sintieron capaces de abordar la escritura de textos académicos, que es posible replicar esta experiencia y que se ha sistematizado la asignatura de Propuesta de Proyecto basada en fundamentos científicos.

\section{Abstract}

This article is derived from the action research project conducted during the course "Reading and Writing Across the Curriculum in Higher Education" offered by the Literacy Program directed by the Academic Center of Excellence for Research and Dissemination of Reading and Writing in PUCMM. The purpose of this research was to study the teaching and learning of writing on the subject Proposal Project Degree in Architecture. Data were collected during the September December 2013 term, which aimed to identify strategies that facilitate the improvement of academic writing in seniors Architecture students. The main conclusions were that knowing the process of writing and the elements of discourse, the students were able to approach the writing of academic texts, that it is possible to replicate this experience and that the subject Project Proposal has been systematized based on scientific foundations.

\section{Palabras claves}

investigación-acción; alfabetización académica; escritura académica; estudiantes universitarios; enseñanza aprendizaje; tesis de grado de arquitectura.

\section{Keywords}

action research; academic literacy; academic writing; university students; teaching learning; architecture thesis.

\footnotetext{
* Orisell Medina Lagrange: Arquitecta, Magister en Arquitectura Tropical Caribeña; Especialista en Pedagogía Universitaria de la Pontificia Universidad Católica Madre y Maestra; candidata a Doctora en Arquitectura y Urbanismo por la Universidad del Bío Bío, Chile. Profesora a tiempo completo del Departamento de Arquitectura PUCMM CSTA. Para contactar a la autora: o.medina@ce.pucmm.edu.do y orisellml@gmail.com
} 
Partiendo de la experiencia docente en el área de Investigación del Proyecto de Grado en Arquitectura desde el año 2008 en la PUCMM, se constató la dificultad de los estudiantes al afrontar la fase de escritura de sus proyectos de grado, lo cual entorpece el desarrollo del proceso de investigación y su posterior concreción en el proyecto arquitectónico. La asignatura forma parte del último año de estudio y es la primera de las tres asignaturas en las que se realiza el Proyecto de Grado:

\section{Gráfico 1. Estructura del Proyecto de Grado de Arquitectura PUCMM CSTA}

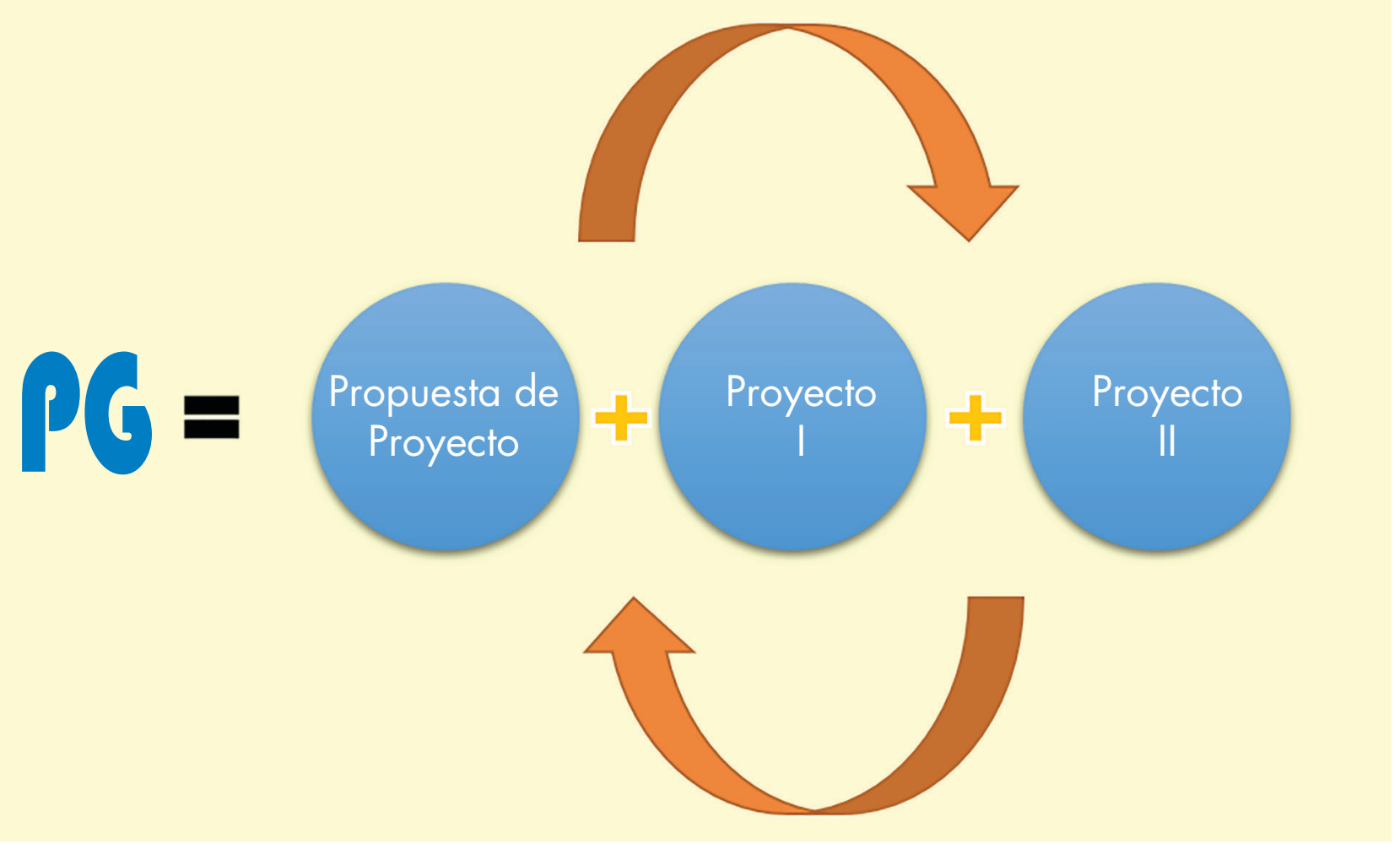

Esta primera etapa constituye la fase de identificación, investigación y fundamentación teórica del Proyecto de Grado. Son definidos el tema, el desarrollo del marco teórico y el análisis de investigaciones similares realizadas en la actualidad; al final de esta se llega a plantear una alternativa de proyecto arquitectónico donde se realice la traducción de la respuesta teórica dada por el estudiante al problema planteado como inquietud de investigación (a desarrollar en su totalidad durante Proyecto I y II).

Entonces, a sabiendas de que la escritura de un documento académico genera ansiedad en los estudiantes, al no estar acostumbrados a leer y a escribir académicamente, diversos autores plantean que en el nivel superior es imprescindible la adopción de estrategias que fomenten la comprensión y el aprendizaje de textos para que los estudiantes desarrollen estrategias metacognitivas en los procesos de lectura y escritura.

En ese orden, Carlino (2012) sostiene que lo que esperan las comunidades académicas universitarias no es lo mismo que los estudiantes han aprendido previamente, puesto que éstas son "nuevas formas discursivas" (p. 23) que desafían y derriban al estudiante si el docente no está preparado para ayudarles a superarlo; para esto se sustenta en Flower y Hayes (1996) quienes plantean que "escribir, para los estudiantes es un acto de cruzar fronteras, o de pararse en el umbral tratando de imaginar cómo cruzar". Carlino (2012) apunta que es preciso hacerse cargo de la lectura y la escritura en cada asignatura para que los estudiantes construyan conocimiento, pues esto es una forma de enseñar estrategias de aprendizaje que acompañarán a los estudiantes por siempre.

Para Cassany (2011), "la escritura es la habilidad lingüística más compleja" (p. 39), pues no basta redactar, sino también tener habilidades para leer, comprender textos y expresarlos de manera oral. En este escenario se hace entonces necesario trabajar para construir una estructura que sirva de soporte al desarrollo de estas competencias. Atendiendo a los procesos de transformación escriturales, Esteban, Mantelli \& Sivori (2009) apuntan que promover la escritura mediante la producción de textos que se van reformulando, es dar un nuevo sentido a la reescritura, plantean que es preciso adoptar este enfoque para contrarrestar la creencia del "espontaneísmo de la escritura y en consecuencia su falta de revisión" (p. 289). 
Por esto Carlino \& Martínez (2009) insisten en que el proceso de lectura y escritura debe ser enseñado dentro de las mismas disciplinas y sus contextos, "para potenciar la experiencia desde el mismo campo disciplinar" (p. 14-15), teniendo como soporte políticas institucionales en ese sentido.

Escribir una tesis de grado se ha revelado como el mayor reto de la preparación en el primer escalón del nivel superior. Implica poner a prueba las competencias adquiridas por los estudiantes durante los años formativos. Carlino (2009) sostiene que si se prevén dispositivos, estrategias y recursos institucionales, es posible que los estudiantes afronten esta etapa con éxito, mientras Graves (1996) afirma que para enseñar a escribir es preciso dominar el arte de la escritura y el de la enseñanza, pues "ningún profesor que no se haya ejercitado en la escritura puede enseñar de manera eficaz el arte del escritor" (p. 23). Cassany (2012) sostiene que el "escribir no es una habilidad espontánea como conversar" (p.19), pues es preciso realizar operaciones constantes y complejas de reflexión y corrección de lo escrito, ya que es necesario releer, corregir y reformular repetidamente lo que se está escribiendo.

Según los planteamientos de Sanjurjo \& Vera (2003), la labor docente en el ámbito de la comprensión y producción de la escritura adquiere una dimensión mayor cuando, partiendo de su propia formación profesional, esta es entendida como un proceso perfectible, que le permita llegar a un grado de conocimiento experto. Estas autoras destacan como fundamental que los docentes hagan de su práctica un objeto de estudio, que tomen distancia para poder reflexionar de manera crítica sobre ella al incorporar nuevos "cuerpos teóricos" (p. 13) y puedan modificar o enriquecer su experiencia en un proceso recursivo. Esto conlleva a observar la enseñanza y aprendizaje de la lectura y escritura académicas, como fases de alta complejidad que requieren ser abordadas con conocimientos sólidos y desde una perspectiva que contemple la autorevisión y perfeccionamiento de la práctica docente.

Para entender el proceso de composición y comprensión de textos académicos, es preciso abordar unas premisas fundamentales, que Díaz-Barriga \& Hernández (1999) plantean en cinco fases y que es necesario sean un punto de partida para la reflexión de las concepciones que tienen los docentes sobre los procesos de producción de escritura en la academia:

\section{Gráfico 2. Premisas del proceso de comprensión y composición de textos}

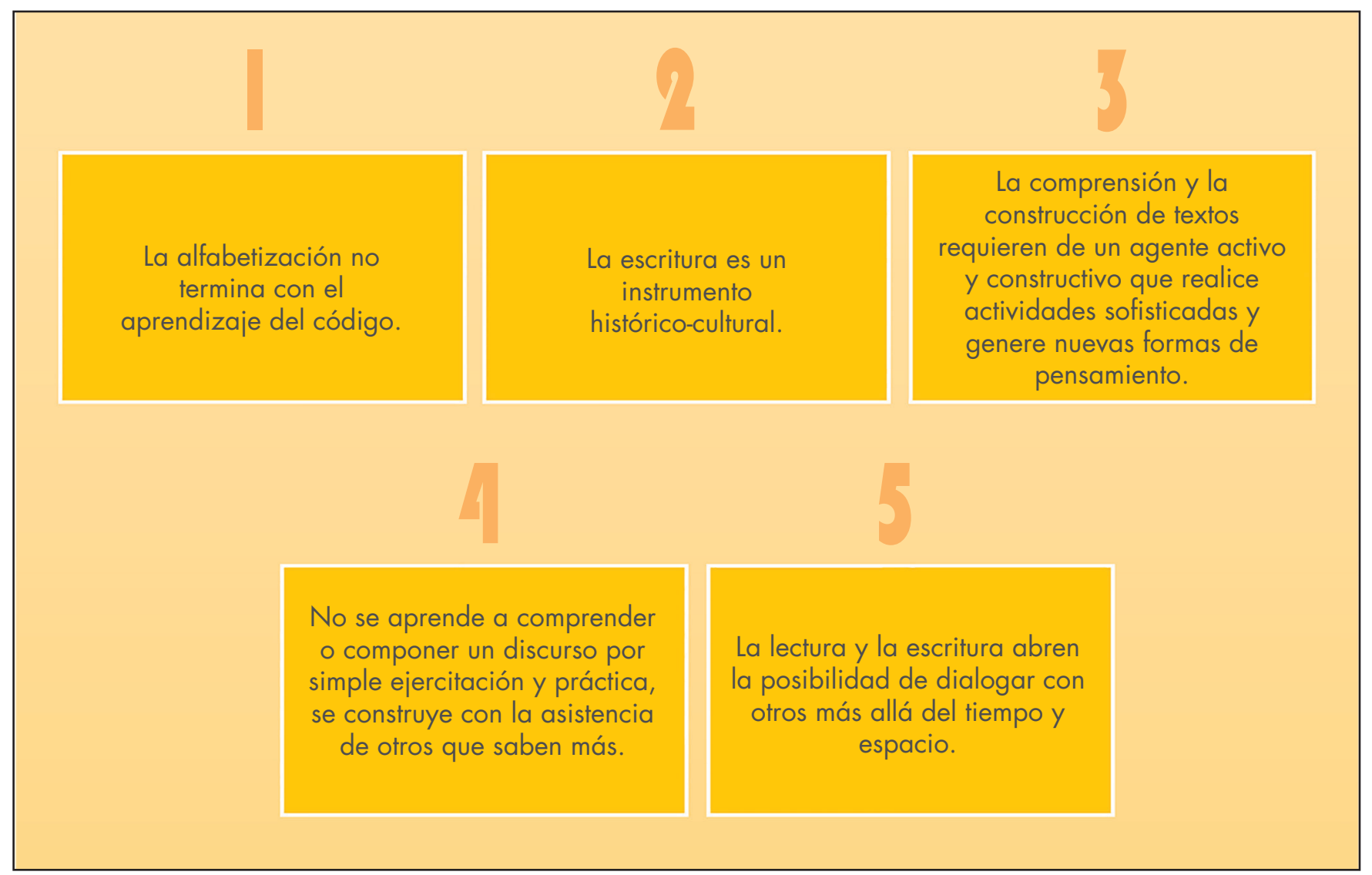

Fuente: Medina, O. (2013), en base a Díaz-Barriga \& Hernández (1999). 
Dichas premisas, según Díaz-Barriga \& Hernández (1999), apuntan a que es conveniente plantear un conjunto de estrategias, que dividen en dos grupos: estrategias de comprensión de textos y estrategias de composición de textos. Cada una de estas, integradas en el proceso de enseñanza aprendizaje, aportan a la construcción de un proceso cognitivo complejo.

El proyecto de investigación-acción realizado en esta asignatura es novedoso en cuanto se basa en no trabajar exclusivamente con una sola estrategia, sino que tiende a sistematizar un conjunto de estas y modelarlas para comprobar si la incorporación de las mismas contribuirían al fin último de esta investigación.

Durante la experiencia docente en Propuesta de Proyecto de Grado ha estado latente la inquietud entre los docentes que la imparten sobre cuáles procesos pudieran dar lugar a que se geste una transformación en cómo los estudiantes abordan esta etapa inicial de último año de carrera. Buscando respuestas a esta interrogante, el objetivo que fundamenta esta investigación-acción es el siguiente: mejorar el desempeño académico de estudiantes de término de Arquitectura mediante el desarrollo de procesos cognitivos y metacognitivos en la producción escrita de la tesis de grado.

\section{Justificación del estudio}

Partiendo del programa de la asignatura, la dirección del Departamento de Arquitectura facilitó algunos documentos básicos a modo de guía general de las etapas de desarrollo del Proyecto de Grado. Los profesores han ido construyendo con base en esto sus criterios del proceso de la Propuesta de Proyecto.

En ese tenor, desde el Departamento de Arquitectura, se llegó a un acuerdo entre los profesores de la asignatura para reforzar esta primera fase, distribuyendo algunas guías básicas de escritura, pues los productos escriturales de las tesis de grado, en muchos casos, evidenciaban las debilidades en la articulación de las ideas y la expresión de las mismas, a través del texto académico que debe ser el documento final de disertación del proyecto. A pesar de estos intentos, no existía una forma sistematizada de impartir la asignatura, y es a partir de cursar la Especialidad en Pedagogía Universitaria, que comienzo a conocer y aprender estrategias de cómo sistematizar los procesos de investigación en las asignaturas.

Se realizó este proceso a modo de prueba en el año 2011, ajustándolo al nivel de los estudiantes; en esa ocasión se obtuvieron documentos con un mejor grado de coherencia en cuanto a la comunicación escrita de las ideas.
En el año 2012 fue evolucionando la forma propia de impartir la asignatura, afinándola a partir de la experiencia anterior, pero era preciso llevar el proceso a un nivel más complejo y encontrar la manera de unirlo a estrategias de lectura y escritura para que los estudiantes llegaran a la metacognición y que a traves de esto los mismos puedan construir conocimientos fundamentales para que mejoren su desempeño en la escritura académica.

En el año 2013 y gracias al Diplomado de Lectura y Escritura a través del Currículo en el Nivel Superior, se consolidó una metodología con estrategias específicas cognitivas y metacognitivas que facilitaron no solo la comprensión, sino también que contribuyeron en la mejora de la capacidad de autoregular el aprendizaje; todo esto derivó en el perfeccionamiento del proceso.

\section{Método}

Este proyecto de investigación-acción tomó lugar en el período académico 1-2013-2014, en el campus Santo Tomás de Aquino.

El enfoque principal de esta investigación consistió en mejorar los procesos de lectura y escritura dentro de la investigación del proyecto de grado, para que pudieran darse procesos metacognitivos en los estudiantes, tratando de despejar la bruma que tradicionalmente envuelve al proceso de realización de la tesis.

Se trabajó el programa de la asignatura integrando estrategias y actividades que facilitaran la consecución del propósito de la investigación, proceso que se reveló muy arduo, pues fue preciso modelar y ajustar cada una de éstas cuando no daban el resultado esperado.

En el planteamiento de esta investigación se utilizó un acercamiento metodológico de investigación-acción, que parte de una estructura sistematizada y perfectible, pues tuvimos la oportunidad de ir perfeccionando o incluyendo estrategias que se consideraron oportunas durante el proceso.

La investigación-acción o action research, para su creador Lewin (1944) en Castillo \& Cabrerizo (2006) conlleva un proceso de "carácter participativo, impulso democrático, y contribución simultánea al cambio y a la conciencia social” (p.169-170). Está pensada como una forma específica de intervención en entornos concretos.

Se utilizó un acercamiento metodológico basado en la participación activa, crítica, colaborativa y constructiva de los estudiantes y sus procesos escriturales como objeto de estudio. 
Para Castillo \& Cabrerizo (2006), el método de investigación-acción persigue un aprendizaje centrado en y desde la práctica, teniendo una dimensión reflexiva, pues todo proceso de investigación-acción "está relacionado con el modo en que se concibe la enseñanza y se desarrolla el currículum académico" (p.170).

Para Medina Rivilla (1991) como se cita en Castillo \& Cabrerizo (2006) esto está basado en:

- Identificación del problema.

- Análisis del problema.

- Formulación de hipótesis-acción.

- Acción y control.

- Evaluación de los cambios, instrumentos y procedimientos.

\section{Participantes}

Los estudiantes participantes en esta investigación de la asignatura Propuesta de Proyecto de Grado, se encontraban cursando el inicio del último año de la carrera de Arquitectura, estaban divididos en dos grupos:

1. el grupo 005 conformado por dos hombres y tres mujeres,

2. y el grupo 006 por un hombre y cuatro mujeres, con edades comprendidas entre los 22 y 23 años de edad.

En 5 sesiones del proceso se contó con el acompañamiento de una experta en lingüística del Centro de excelencia para la investigación y difusión de lectura y escritura (CEDILE), la cual observaba las actividades y colaboraba en el modelado de las estrategias que así lo necesitasen.

\section{Procedimiento e instrumentos}

Las estrategias consideradas pertinentes para esta investigación fueron desarrolladas desde los ejercicios realizados en el contexto del Diplomado de Lectura y Escritura en el Nivel Superior y replicadas (ajustadas o modificadas) en la asignatura de Propuesta de Proyecto de Grado.

Las estrategias utilizadas durante esta investigación (al realizar una secuencia desde el año 2008 al 2013) aparecen representadas en el gráfico 3 , de la siguiente manera:

\section{Gráfico 3. Línea de tiempo de las estrategías utilizadas}

Proceso ordenado (método científico) sin estrategias especializadas

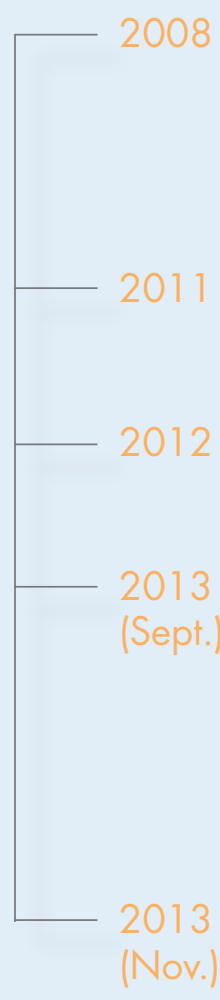

Tabla de la inquietud

Ficha bibliográfica

Tabla de la inquietud

Ficha bibliográfica

Tabla de la inquietud

La lista

Ficha bibliográfica

Presentaciones audiovisuales

Heteroevaluación

Tabla de la inquietud

La lista

Análisis de discurso

Ficha bibliográfica modificada

Transformar el conocimiento en

discurso académico- Operadores del

discurso

Coevaluación

Para texto

Heteroevaluación

Las estrategias de enseñanza aprendizaje integradas al proceso de escritura de las tesis de grado en Arquitectura consideradas pertinentes fueron:

\section{Tabla de la inquietud}

Esta estrategia fue integrada a partir de la experiencia en Proyecto Pedagógico I de la Especialidad en Pedagogía Universitaria, y se utilizó esta tabla (diseñada por la Lic. Marta Vicente) y ajustada por la investigadora para el nivel de pregrado.

Aquí los estudiantes comienzan a describir de manera estructurada, indagando las inquietudes que tienen dentro del campo de la Arquitectura para determinar cuáles pueden ser investigables, para seleccionar en un proceso de descarte, la que primará en el desarrollo de la Propuesta de Proyecto. La evaluación de esta estrategia de inicio consistió en constatar el grado precisión y pertinencia en la selección de una inquietud de investigación. El instrumento es el siguiente: 


\section{LA INQUIETUD \\ PONTIFICIA UNIVERSIDAD CATÓLICA MADRE Y MAESTRA - CSTA \\ FACULTAD DE CIENCIAS Y HUMANIDADES \\ DEPARTAMENTO DE ARQUITECTURA}

\section{Propuesta de Proyecto, ARQ-418-T \\ 1-2013-2014}

Nombre:

PRIMERA SESION- 30 de agosto y 4 septiembre 2013

1. Práctica $\# 1^{2}$. Primera aproximación a la pregunta de investigación: La curiosidad

"Un viaje de mil millas comienza con el primer paso." Lao - Tse

Esta asignatura está estructurada sobre la base de construir de forma clara y pertinente la etapa de identificación de la inquietud y la consecuente investigación del proyecto de grado. Las prácticas giran en torno a la construcción, de manera concéntrica, de los procedimientos necesarios para formular la o las ideas sobre la cual se pueda estructurar la investigación.

En particular, en esta práctica \#1 se busca trabajar con las primeras inquietudes de los estudiantes para desmenuzar y sacar a la luz ideas que sirvan como semillas que permitan el desarrollo del proceso.
Iniciamos siguiendo esta estructura:

1. Escribir el nombre en el encabezado de la Tabla 1.

2. Transcribir las inquietudes (máximo cuatro) que tengan en torno a la Arquitectura en la primera columna de la Tabla 1. Mejorar la expresión de la idea si es preciso y añadir nuevas inquietudes que hayan surgido a partir de la clase anterior.

3. Escribir con más detenimiento, en la segunda y tercera columna de la Tabla 1, el por qué de esa inquietud y qué se quisiera saber a partir de un proceso investigativo que surja de esa inquietud. Veamos la Tabla 1.

\section{Tabla 1}

\section{Nombre:}

\begin{tabular}{|l|l|l|}
\hline Inquietudes & ¿Por qué pienso así? & ¿Qué quisiera averiguar? \\
\hline
\end{tabular}

4. Determinar, en la Tabla 2, a qué tema pertenece cada inquietud, con cuáles otros sub-temas se relaciona y con qué Proyectos de grado ya terminados tiene alguna similitud.

La información de esta columna será útil también en prácticas sucesivas. Su función en esta práctica es tomar conciencia del nivel en que esa inquietud ha sido compartida, trabajada y puesta al acceso público por otros estudiantes de la PUCMM CSTA. El listado de los Proyectos de Grado está disponible en el Departamento de Arquitectura y en la oficina de la Prof. Orisell Medina en el B1-T-N. 


\section{Tabla 2}

Nombre:

\section{Inquietudes}

5. Ordenar cada inquietud en la Tabla 3 según los criterios expuestos a continuación. Aunque resulte difícil jerarquizar un orden, intentar forzarlo, de manera que las ideas adquieran más claridad. Se asignarán valores de 1 a 5 , siendo 1 la puntuación más alta (criterio por orden de importancia).

A. Interés y gusto por el tema.
B. Intuición sobre la posibilidad de que la inquietud sea investigable.

C. Intuición sobre la posibilidad de compartir esta inquietud con otro(s) compañero (s) de clase para trabajar juntos .

D. Pertinencia de la inquietud para mejorar mis conocimientos y comprensión.

\section{Tabla 3}

\section{Nombre:}

Inquietudes
Criterios

\begin{tabular}{l|l|l|l} 
A & B & C & D
\end{tabular}

6. Depurar en la Tabla 4 sólo las casillas a las que se le asignó el número 1.

\section{Tabla 4}

\section{Nombre:}

\section{Inquietudes}


7. Concluir, a partir del proceso realizado en los puntos 5 y 6 , cuál inquietud prevalece sobre las demás. Escribir en la Tabla 5 un máximo de 5 razonamientos que llevan a esta conclusión. El total de palabras de la Tabla 5 no debe exceder de 200. Para verificar este número, usar la herramienta "Word Count" o "Conteo de Palabras" de Word.

\section{Tabla 5}

Nombre de la inquietud:

Razones por las que prevalece sobre las demas:
1. Una
2. Dos
3. Tres
4. Cuatro
5. Cinco

\section{La lista de referencias}

La lista está sustentada en las ideas iniciales de Golde (2010) como se cita en Moreles \& Jiménez (2012), quienes la plantean como una estrategia que parte del supuesto de que conocer la bibliografía o estado del arte, relativo a la inquietud y temática que el estudiante ha decidido abordar como proyecto de investigación teórica para la tesis de grado, le permitirá desarrollar un conocimiento claro del tema y con la suficiente información que sirva de sustento conceptual a su proyecto arquitectónico. La lista es el programa de lecturas que se desarrolla con y para la tesis.

Para Golde (2010) como se cita en Moreles \& Jiménez (2012) demostrar el dominio del tema en cuestión requiere que el estudiante-investigador lea y analice críticamente las obras escritas de los principales autores del área temática seleccionada. La lista alimenta en primera instancia a las fichas bibliográficas (explicadas más adelante) y es el instrumento que sirve para ordenar la información.

Consiste en una tabla donde cada estudianteinvestigador coloca los textos en el orden jerárquico en que serán leídos. Esta estrategia no se evaluó cuantitativamente, sino al ser utilizada como mapa de ruta o guía en el proceso de investigación, el uso de los autores referentes se iría verificando en las entregas de informes de avance. Se utilizó el siguiente instrumento elaborado a partir del modelo de Golde (2010):

\section{Tabla 6: Lista de referencias}

\section{LA LISTA DE REFERENCIAS \\ PONTIFICIA UNIVERSIDAD CATÓLICA MADRE Y MAESTRA - CSTA \\ FACULTAD DE CIENCIAS Y HUMANIDADES DEPARTAMENTO DE ARQUITECTURA}

Propuesta de Proyecto, ARQ-418-T
1-2013-2014

Nombre del estudiante:

\section{La Lista 2013. Lista de Referencias.}

"Lee y conducirás, no leas y serás conducido". Santa Teresa de Jesús.

Presentamos "La Lista" (Golde, 2010), estrategia que trabajaremos en esta asignatura partiendo del supuesto de que al conocer la bibliografía o "estado del arte", relativo a la inquietud y temática que el estudiante ha decidido abordar como proyecto de investigación teórica para la tesis de grado, les permitirá desarrollar un conocimiento claro del tema y con la suficiente información que le sirva de sustento conceptual a su proyecto. La lista es el programa de lecturas que se desarrollará con y para la tesis.

Para Golde (2010), demostrar el dominio del tema en cuestión requiere que el estudiante-investigador lea y analice críticamente las obras escritas de los principales autores del área temática seleccionada. La lista alimenta en primera instancia a las fichas bibliográficas y es el instrumento que nos servirá para ordenar la información. Consiste en una tabla donde cada estudiante-investigador colocará las obras en el orden jerárquico en que serán leídas. 
La Lista contiene las siguientes informaciones (cada quien agregará tantas filas como sean necesarias).

\section{Análisis del discurso}

La estrategia de análisis del discurso fue incluida a partir de conocer los elementos que componen a las comunidades discursivas y las implicaciones del discurso como hecho comunicativo. Van Dijk (1980) argumenta que dentro de las comunidades lingüísticas las reglas convencionales de comunicación son conocidas implíci tamente por sus miembros, pero que se precisa que sean explicitadas para una mejor comprensión dentro de la comunidad discursiva. Para ampliar la comprensión del concepto, la investigación se ha apoyado en lo expresado por Bajtín (1979) citado por García \& Negroni (2001), donde se plantea que el discurso se llena de entonaciones anteriores, de voces cuyos miembros están en pleno diálogo.

Este autor establece que todo enunciado es un eslabón y cuenta con enunciados anteriores con los cuales establece toda suerte de relaciones (se apoya en ellos, polemiza con ellos, o simplemente los supone conocidos por su oyente). Esto fue fundamental para que los estudiantes comprendieran y reconocieran las voces dentro de los textos y el peso de cada una de ellas en la comprensión del discurso de los autores referentes.
En este caso se utilizaron textos cortos para identificar las voces discursivas y el rol que jugaban dentro del acto comunicativo en cuestión, para luego ser aplicado este análisis a la comprensión de los textos referentes del estado del arte de cada estudiante. Al igual que la estrategia anterior la evaluación se verificó en las entregas de avance al ir revisando si el estudiante integraba los conocimientos adquiridos a su proceso escritural.

\section{La ficha bibliográfica}

La ficha bibliográfica surge a partir de las fichas temáticas planteadas por Cifuentes (2011), quien las define como un extracto donde están contenidas las informaciones seleccionadas del tema investigado y a la producción de conocimiento sobre la temática del proyecto de investigación; en ella se plasma el análisis escrito de los textos leídos. La intención es que el estudiante realice una revisión de la literatura a profundidad para fundamentar conceptualmente su proyecto de investigación de grado; la finalidad es buscar en los textos referenciales y complementarios el sentido y la pertinencia de éstos en el problema que se plantearon. El instrumento utilizado fue ampliado a partir del realizado por la Lic. Marta Vicente, del cual se presentan dos extractos:

\section{Gráfico 4. Fichas bibliográficas utilizadas, reelaboradas a partir de Vicente (2011) y Cifuentes (2010)}

Tabla 1: Tabla Ficha Tipo A

\begin{tabular}{|c|c|c|c|c|c|}
\hline \multicolumn{6}{|c|}{ Pregunta que prevalece: } \\
\hline \multicolumn{6}{|c|}{ Origen del texto: } \\
\hline Autor & Año & Título & \multicolumn{2}{|c|}{ Editorial o institución editora } & Tipo de publicación \\
\hline \multicolumn{6}{|c|}{ Razón para seleccionar esta bibliografía } \\
\hline \multicolumn{2}{|c|}{\begin{tabular}{|l|} 
Fragmento de texto \\
\end{tabular}} & Síntesis de las ideas recogidas & Palabras claves & \begin{tabular}{|l|} 
Veces en el discurso \\
\end{tabular} & $\begin{array}{l}\text { Interpretación del investigador sobre los } \\
\text { aportes de la información analizada }\end{array}$ \\
\hline
\end{tabular}

Tabla 2: Tabla Ficha Tipo B

\begin{tabular}{|l|l|l|l|l|}
\hline Pregunta que prevalece: \\
\hline Origen del texto: \\
\hline Autor & Año & Título & & \\
\hline
\end{tabular}

Esta estrategia fue de la mano con la del análisis del discurso, pues se pedía explícitamente que integraran dicho análisis en las fichas para luego introducirlas en el informe de avance. 


\section{0 • VENTANAS ABIERTAS A LA PEDAGOGÍA UNIVERSITARIA}

\section{Organizadores gráficos}

En la asignatura, se utilizó la estrategia de comprensión de textos expositivos a través de los organizadores gráficos, aprovechando la facilidad que tienen los estudiantes de arquitectura para generar información de carácter visual.

En ese sentido, Padilla, Douglas \& López (2010) plantean que el hecho de extraer las ideas fundamentales de un texto implica construir uno nuevo partiendo del texto base, pues de acuerdo al propósito del lector, éstas pueden ser reorganizadas y jerarquizadas (en este caso de manera gráfica), se propuso el análisis de textos para que fueran explicados mediante las estrategias del mapa conceptual y el esquema conceptual.

La producción de mapas conceptuales como medio de elaboración de conocimiento es señalada por Arellano \& Santoyo (2009) quienes plantean que por medio de la comprensión de conceptos fundamentales y los vínculos asociados a ellos, se pueden abstraer y generar nuevos conocimientos.

Con la utilización de esta estrategia se pudieron recuperar saberes a partir de la creatividad, aplicada al análisis y comprensión de un texto, mostrando el trabajo realizado al interpretar autores a través de gráficos para enriquecer y aportar dinamismo al texto que se estaba desarrollando.

\section{Transformar el conocimiento en discurso académico-operadores del discurso}

Padilla, Douglas \& López (2010), hacen referencia a lo planteado por Scardamaglia \& Bereiter (1992) en cuanto a que existen dos modelos explicativos del proceso de producción escrita:

1. Decir el conocimiento.

2. Transformar el conocimiento.

Lo primero sucede a los escritores novatos que se limitan a reproducir lo que otro ha dicho: no hay planificación antes de escribir, no se tiene en cuenta al posible lector y, al revisar el texto, no hay transformación sustancial del contenido.

A diferencia de lo segundo, que ocurre cuando el escritor experto realiza una actividad de escritura muy estructurada y sofisticada, "lo que precisa de una reflexión sobre qué se va a incluir en un texto concreto y cómo va a escribirse" (Scardamaglia \& Bereiter, 1992), en Padilla, Douglas \& López (2010), p.62-63.
Esta manera de abordar la escritura permite comprender que para pasar a procesos de metacognición, el estudiante "escritor novato" necesita de un andamiaje para convertir su proceso escritural en el de un "escritor experto".

Los operadores del discurso son definidos por Abarca (2012) como "unidades cuya función consiste, de manera explicita, en relacionar segmentos de un discurso" (p. 144). Se planteó un primer ejercicio sobre los operadores del discurso donde cada estudiante debía llevar a la clase cinco frases con las cuales uno de los autores referenciales de su tema diera inicio a una cita.

A seguidas, formados en grupos, seleccionaban las cinco que consideraran ideales para citar y al final se creaba una lista común de operadores que se añadió a la lista que había construido el Departamento de Arquitectura dentro de los documentos básicos de soporte a Propuesta de Proyecto, se pidió que fueran incorporadas en el siguiente informe de avance.

\section{Coevaluación}

Una de las actividades más polémicas en el proceso de enseñanza aprendizaje es la evaluación y este ámbito conflictivo fue convertido en un espacio para la observación, la crítica constructiva y el diálogo entre los estudiantes del curso Propuesta de Proyecto.

Se utilizó la coevaluación, definida por Barros, Iranzo, Soler \& Tierno (S/F) como un proceso donde se reflexiona y se discute a lo largo del desarrollo de las actividades del grupo, incorporándola como parte del proceso formativo. Esto ocurre al establecer previamente los criterios de evaluación, para lograr una participación con más conciencia de lo que se buscaba en el proceso de enseñanza aprendizaje, emitir juicios críticos y proponer opciones de mejora.

Adoptando un enfoque más humanista en las posibilidades didácticas de la evaluación, centrada en el trabajo colaborativo y en principios éticos sobre los niveles de tolerancia al estilo de producción escrita de cada quien, se utilizó esta estrategia, haciendo referencia a Cassany (2012) quien apunta que siendo la enseñanza un proceso colaborativo de desarrollo personal, olvidando los antiguos esquemas del "profesor como pozo de ciencia absoluta" (p. 46) o de traspaso de información unidireccional profesor-estudiante, estos últimos pueden convertirse en "auto correctores y en correctores de los trabajos de sus compañeros" Cassany (p. 46).

El instrumento de coevaluación diseñado por la Lic. Florilena Paredes, acompañante del proceso, para este curso fue el siguiente: 


\title{
LA COEVALUACIÓN. RUBRICA COEVALUACIÓN DE UN TEXTO ACADÉMICO PONTIFICIA UNIVERSIDAD CATÓLICA MADRE Y MAESTRA- CSTA FACULTAD DE CIENCIAS Y HUMANIDADES DEPARTAMENTO DE ARQUITECTURA
}

\author{
Propuesta de Proyecto, ARQ-418-T
}

1-2013-2014

\section{Evaluador:}

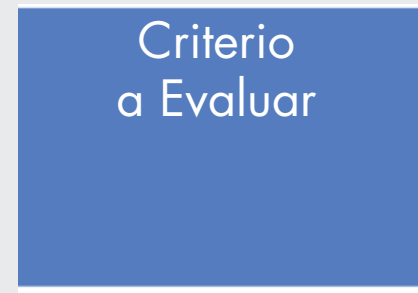

Selección del material relevante para el desarrollo del texto

La calidad, originalidad y coherencia del análisis llevado a cabo

\section{Dominio razonable del tema}

Capacidad de comunicación y estilo

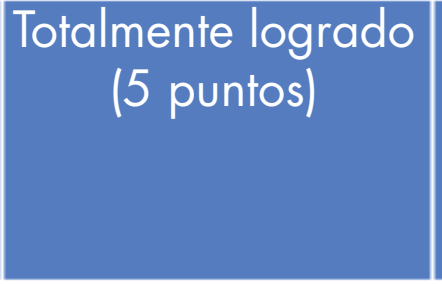

Muestra un conocimiento amplio de la literatura o teorías seleccionadas. Identifica puntos de contacto o diferentes entre autores. Presenta una postura crítica respecto a los autores de referencia. La literatura está ordenada en base a un criterio. Presenta una justificación de por qué esta es la literatura relevante.

El texto posee calidad en su presentación, organización y coherencia y logra los objetivos propuestos. Sus ideas son claras y presentan un análisis profundo del tema. Su tema es original.

El texto demuestra un gran dominio, ya que presenta el 'estado del arte' en el tema, incluyendo desarrollos actuales, controversias $\mathrm{y} / \mathrm{o}$ avances. Tiene una bibliografía justa y se muestra en el texto a través de las citas e intertextualidad con otros autores, aunque con una postura crítica y analítica.

Demuestra una hilación clara de las ideas, es interesante y posee un estilo atractivo. Tiene expresividad y una cohesión adecuada.

\section{Evaluado:}

\section{Medianamente logrado (3 puntos) (O solo presenta tres de los criterios de la columna de la izquierda)}

Muestra un conocimiento amplio de la literatura - teorías seleccionadas. Identifica puntos de contacto o diferentes entre autores y la literatura está ordenada en base a un criterio.

El texto posee calidad en su presentación, organización y coherencia y logra los objetivos propuestos, pero sus ideas son ambiguas y su redacción es poco clara. El tema es original, pero presenta un análisis poco profundo.

El texto demuestra un gran dominio, ya que presenta el 'estado del arte' en el tema, incluyendo desarrollos actuales, controversias $\mathrm{y} / \mathrm{o}$ avances. Pero presenta excesos o ausencia de citas cuando corresponde, y cuando aparecen, no plantea su postura ante lo dicho por los otros autores.

Demuestra una hilación clara de las ideas, pero su estilo es periódico provocando la pérdida de interés en el lector. En algunas ocasiones no se escogieron los términos más adecuados y hay mucha repetición de los mismos.

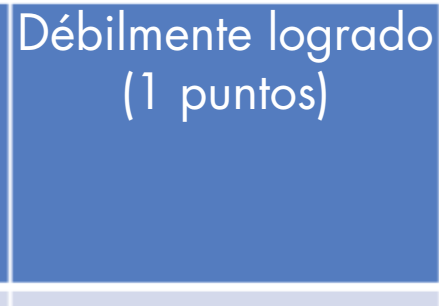

Presenta un listado de literaturas o teorías seleccionadas. Identifica puntos de contacto o diferentes entre autores, pero la literatura no está ordenada en base a un criterio.

El texto posee poca calidad en su presentación. Su organización y coherencia son débiles y no logra los objetivos propuestos.

El texto demuestra un débil dominio del tema, no incluye desarrollos actuales, controversias $y / o$ avances. Su bibliografía es "inflada", ya que no aparecen esas citas dentro del texto.

No hay una presentación clara de las ideas. Su estilo es periódico provocando la pérdida de interés en el lector, - deja ideas inconclusas. En algunas ocasiones no se escogieron los términos más adecuados y hay mucha repetición de los mismos.

El estudiante resaltará en amarillo en esta rúbrica, los elementos descritos que encuentre dentro del texto a revisar. Comentarios: 


\section{2 - VENTANAS ABIERTAS A LA PEDAGOGÍA UNIVERSITARIA}

\section{El paratexto}

Según el portal Educar.ar el paratexto es:

El primer contacto del lector con el material impreso y, desde este punto de vista, funciona como un instructivo o guía de lectura, ya que le permite anticipar cuestiones como el carácter de la información y la modalidad que esta asumirá en el texto. (Párrafo 1).

El paratexto fue planteado al final del curso para que los estudiantes distinguieran los elementos que componen un texto impreso y pudieran usar estos conocimientos y criterios en la diagramación final de su libro de Proyecto de Grado, teniendo como premisa básica que el paratexto es todo lo que rodea o acompaña al texto.

Se analizó un libro como ejemplo general y cada estudiante hizo lo mismo para ir identificando los elementos paratextuales en un documento de su lista de referencias. A partir de este ejercicio, los estudiantes diseñaron dos páginas guías para su documento final de grado. A modo de evaluación se realizaron comentarios del grupo para cada participante y su propuesta de diagramación.

\section{Heteroevaluación}

A partir del modelo de Guía-cooperación de Stelzer Morrow (1991) en Cassany (2012), se realizaron observaciones tanto de fondo como de forma donde el profesor marca los errores, orienta la corrección y el alumno sigue las instrucciones.

Esto fue realizado a través de símbolos y comentarios, con el objetivo de no darles la solución al error, sino que ellos pensaran al revisar y reescribir los apartados necesarios.

La heteroevaluación fue realizada de manera constante durante el desarrollo de la asignatura, pues la planificación estipulaba una entrega semanal de informe de avance, el cual era revisado, devuelto con las observaciones y las calificaciones correspondientes, también se comentaban en el aula aspectos a rescatar de dichas correcciones. Cada una de las estrategias utilizadas en la asignatura debían ser incluidas por los estudiantes en la secuencia del desarrollo del documento, como se estipulaba, así se podía entonces constatar el nivel de integración de los conocimientos generados por dichas estrategias en el texto académico.

El sistema utilizado para este proceso fue sobre el documento Word digital subido a la plataforma Moodle (nunca se imprimió en papel durante el curso), en este se realizaban los comentarios y observaciones, siempre atendiendo a que el estudiante se cuestionara y tuviera la oportunidad de reflexionar sobre su trabajo escrito, para perfeccionarlo al seguir la pauta de las siguientes entregas programadas. Además, podían ver los trabajos de sus compañeros.

Esta apertura y flexibilidad en cuanto a estar dispuestos a cambiar, modelar y mejorar estrategias que no estaban dando el resultado esperado, hizo la diferencia en el proceso de enseñanza aprendizaje, pues permitió una riqueza de experiencias y resultados que de otra manera, no hubiese sido posible obtener.

\section{Recopilación de la evidencia}

El primer paso para evidenciar los cambios que iban surgiendo en el proceso, se dio en primera instancia a partir de la heteroevaluación semanal de los dos grupos, utilizando el diario docente para registrar el modelado de las estrategias y sus resultados, y en la etapa final de Propuesta de Proyecto, se procedió a construir un instrumento de evaluación cualitativa para indagar las opiniones del grupo sobre las estrategias utilizadas; este último instrumento fue diseñado a partir de los conocimientos adquiridos en la Especialidad en Pedagogía Universitaria. El cuestionario fue colocado en la plataforma Moodle del curso para que los estudiantes lo llenaran y colocaran en un foro. Cabe destacar que el mismo estuvo disponible para descarga luego de publicados los acumulados finales del curso. De diez estudiantes que finalizaron el curso, nueve respondieron el instrumento.

El cuestionario fue estructurado en cuatro apartados:

1. Valoración de los conocimientos adquiridos en la asignatura.

2. Valoración de las estrategias utilizadas en clase.

3. Selección y valoración de la estrategia que más le ayudó a mejorar su nivel de comprensión de textos y la escritura del documento de tesis.

4. Valoración de la utilidad de los conocimientos adquiridos en Propuesta de Proyecto para la escritura y comprensión de textos académicos.

5. Valoración de la utilidad de la Plataforma Moodle en la organización del curso, acopio de documentos e intercambio de información.

A pesar de ser esta una investigación eminentemente cualitativa, se utilizó la valoración en un escala de 1 al 5 , siendo cinco el valor más alto para poder cuantificar 
el grado de las preferencias de los procesos realizados en la asignatura para que complementaran las respuestas escritas por los estudiantes.

\section{Resultados}

En esta oportunidad fueron analizados los apartados 1 , 3 y 4 de la recopilación de la evidencia:

\section{a. Valoración de los conocimientos adquiridos en la asignatura}

La categoría de valoración que predominó fue el aspecto social: que se aprende mejor escuchando y compartiendo las opiniones de los compañeros de curso en el proceso de enseñanza aprendizaje; este enfoque fue planteado por Vygotsky (S/F) (mencionado en Díaz Barriga \& Hernández, 1999) en sus postulados de la corriente sociocultural y el enfoque constructivista de la pedagogía. Salió a la luz en uno de los cuestionarios que: "Fueron momentos enriquecedores, porque aprendí de las ideas y experiencias de mis compañeros, para luego ponerlas en práctica" (Evaluación acápite A, Cuestionario único, estudiante no. 3). Los nueve participantes valoraron los conocimientos adquiridos como positivos.

\section{b. Seleccionar y valorar la estrategia que más le ayudó a mejorar su nivel de comprensión de textos y la escritura del documento de tesis}

Las estrategias utilizadas fueron valoradas positivamente por los estudiantes, pero las preferencias estuvieron marcadas de la siguiente manera:

\section{Gráfico 5. Preferencia de las estrategias implementadas en la asignatura}

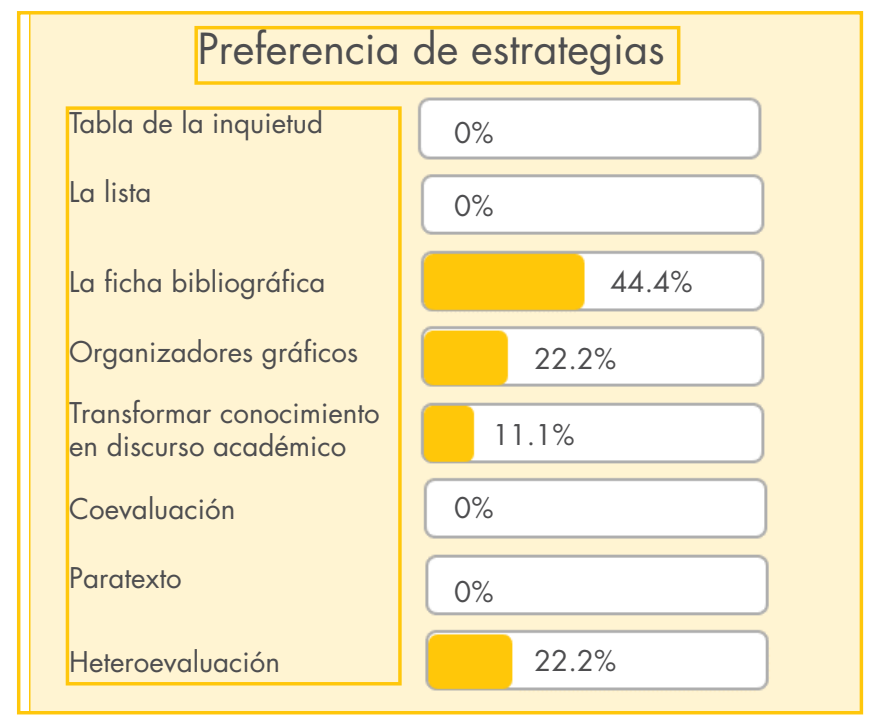

En primer lugar valoraron las fichas bibliográficas, a continuación sus comentarios:

a. "Aunque al principio no encontraba que fuera una herramienta necesaria, luego con el tiempo fui entendiendo su importancia. Simplemente es ir acoplando las ideas que voy escogiendo de cada texto, para así luego poder analizarlas y sacar una conclusión de todo esto. Esa información me ayudó en mi elaboración del marco teórico" (evaluación acápite b, cuestionario único, estudiante no. 1).

b. "Fue una manera rápida y directa de entender las ideas de los autores, para poder interpretarlas y desarrollar el MT" (evaluación acápite b, cuestionario único, estudiante no. 3).

c. "Me permitió sacar la información necesaria de los libros que iba leyendo y trabajarla de un modo que luego me facilitaba la escritura del marco teórico" (evaluación acápite b, cuestionario único, estudiante no. 7).

d. "Porque permite interpretar el mensaje de las distintas voces para poder emitir una opinión propia. Además, ayuda ir respondiendo las preguntas que se habían planteado para la investigación" (evaluación acápite b, cuestionario único, estudiante no. 8).

Cabe destacar que este instrumento, el cual produjo la mayor resistencia a ser utilizado, fue al final el mejor valorado por los estudiantes.

Los estudiantes valoraron en segundo lugar los organizadores gráficos y comentaron respecto a estos que: "Mi investigación fue compleja y extensa debido a la cantidad de información, esto me sirvió de ayuda para la síntesis de ideas importantes" (evaluación acápite b, cuestionario único, estudiante no. 5).

En tercer lugar estuvo el transformar conocimiento en discurso académico, y uno de los estudiantes comentó que lo valoró más porque "al momento de redactar y visualizar la manera en la cual ha sido tratado el tema y los diferentes puntos de vista de quienes han trabajado con el mismo, visualizaba mejor el texto" (evaluación acápite b, cuestionario único, estudiante no. 7). Este acápite fue valorado a la par de la heterovaluación porque "sin duda alguna, esas correcciones sistemáticas con un seguimiento continuo de cómo íbamos avanzando en la redacción hizo que todo lo elaborado tenga un gran peso" (evaluación acápite b, cuestionario único, estudiante no. 4).

\section{c. Valorar la utilidad de los conocimientos adquiridos en Propuesta de Proyecto para la escritura y comprensión de textos académicos}

Este fue uno de los puntos más enriquecedores en cuanto a la experiencia adquirida por todo el grupo. A continuación se presentan los comentarios: 


\section{Tabla 8: Cuestionario único}

\section{Cuestionario único Estudiante No.}

1

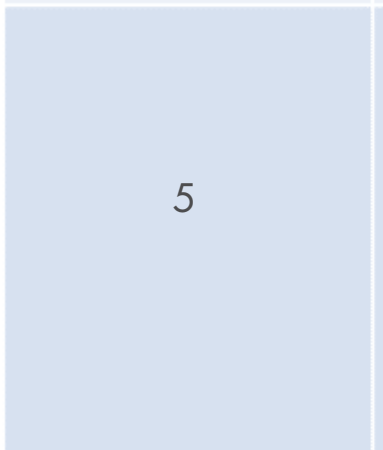

6

La valoración que predominó en base a los comentarios de los estudiantes fue el reconocer que trabajar con sistematicidad y estructura es vital para los procesos de producción escrita en el nivel superior, puesto que al ser conscientes de los elementos

\section{Comentarios}

Encuentro que fue un proceso rápido, pero a la vez fue productivo y ayudó mucho a la redacción, puesto que con esto de las calificaciones y correcciones uno iba viendo sus errores y faltas. Es importante saber plasmar las ideas e interpretar lo que expresan otros autores, y este curso me ayudó.

A medida que vamos adquiriendo más conocimientos sobre cómo redactar un texto correctamente, se va haciendo más y más fácil, al principio se hacía un poco más pesado.

Me convirtió en otro estudiante, es decir me siento confiado al hacer otra investigación, incluso profesionalmente, la investigación siempre es determinante y base firme para la elaboración de cualquier proyecto arquitectónico $u$ otro. Me he comparado disimuladamente con amigos en término, y con compañeros de otras carreras como Mercadeo, Economía, que los fuertes son la investigación y puedo decir que estamos a un nivel superior en este ámbito. Además aprendí una manera diferente al saber capturar lo relevante de un libro para la investigación.

A partir de esta materia, los verbos investigar y escribir cambiaron, ya no son verbos de rutina para la ejecución de esa acción. Se han convertido en herramientas expresivas de conocimientos, hechos desde procesos y metodologías sistemáticas y organizadas que garantizan la calidad de estas acciones.

De ahora en adelante se tiene un manejo a un mayor nivel con respecto a la escritura y los documentos académicos.

El contenido trabajado en Propuesta de Proyecto fue totalmente enriquecedor para mi proceso de escritura del marco teórico, permitiéndome redactar de manera más fácil y clara.

Creo que en Propuesta fue que aprendimos a realmente escribir textos académicos.

Los conocimientos adquiridos sobre escritura y comprensión me hacen sentir como una persona capaz y preparada para poder escribir cualquier tipo de texto académico. Son términos y conocimientos que nos acompañarán por el resto de nuestra vida como profesionales y nos hacen capaces de poder realizar cualquier otra investigación.

Los procesos y la metodología utilizada por la profesora son bastantes oportunos y nos sirven de ejemplo para poder realizar investigación y redacciones de una forma correcta y en el tiempo que amerita. 


\section{Conclusiones}

Esta investigación se planteó responder las inquietudes que surgen del problema enfocado en el proyecto de investigación-acción: ¿cuáles procesos de lectura y escritura pueden ser utilizados en el ámbito de la elaboración de la tesis de grado?, ¿cuáles estrategias de lectura y escritura pueden adaptarse al proceso de investigación?, ¿cuáles estrategias de enseñanza aprendizaje permiten transformar el proceso de producción escrita de los estudiantes hacia la escritura académica metacognitiva? y ¿cómo pueden ser sistematizadas las estrategias para que sirvan de vehículo conductor de la asignatura a nivel departamental?.

Según lo planteado por autores referentes como es el caso de Carlino (2012) y Cassany (2009), es preciso que desde las asignaturas mismas se trabaje con los procesos de escritura académica, incorporando estrategias que faciliten la integración del conocimiento del proceso de producción escritas dentro del currículo, esto fue comprobado durante el desarrollo de esta experiencia, pues al plantearse la necesidad de ir modelando las estrategias y lo que esto implicaba para el desarrollo del curso, pudieron verse los resultados en la mejora de la calidad de los productos escriturales de los estudiantes participantes.

A partir del proceso de análisis se concluye lo siguiente: Los resultados obtenidos en cuanto a preferencias de los estudiantes y sus opiniones apuntan a afirmar que la integración del proceso de lectura y escritura en el nivel superior es viable y realizable dentro de las disciplinas, en cada asignatura, pues hay una diversidad de estrategias que pueden ser modeladas para cada caso. Los estudiantes, al comprender el proceso de la producción escrita y los elementos que componen el discurso, se sienten capaces y preparados para abordar la escritura de los textos académicos.

Es posible replicar esta experiencia en las asignaturas de Proyecto de grado, fundamentándose en los procesos de lectura y escritura en el nivel superior. Mediante este trabajo, se han sentado las bases seguras para avalar la sistematización de Propuesta de Proyecto con fundamentos científicos. Partiendo de la experiencia del Diplomado de Lectura y Escritura a través del Currículo en el Nivel Superior, se entiende que los docentes pueden replicar esta experiencia, si se familiarizan con la investigación-acción y con los fundamentos de la lectura y la escritura a través del currículo, pues es preciso seguir el rigor científico y estar dispuestos a reaprender para mejorar la práctica docente.

\section{Referencias}

Abarca, C. (2012). Operadores discursivos-pragmáticos en consultas cartománticas. Revista de
Humanidades no. 26, pp. 143-158. ISSN: 07170491

Arellano, J. \& Santoyo, M. (2009). Investigar con mapas conceptuales. Procesos metodológicos. Narcea, S.A.: Madrid.

Barrios, C., Iranzo, P., Soler, A. \& Tierno, J. (S/F). La coevaluación y la autoevaluación dentro del sistema evaluativo de una asignatura de la carrera de pedagogía. $4^{\circ}$ Congreso Internacional de Docencia Universitaria e Innovación (CIDUI): "La competencia docente", en el apartado de 'Sistemas alternativos de evaluación de los aprendizajes'.

Carlino, P. (2012). Escribir, leer y aprender en la universidad. Una introducción a la alfabetización académica. FondodeCulturaEconómica:Argentina.

Carlino, P. \& Martínez, S. (2009). La lectura y la escritura: un asunto de todos/as. Editorial de la Universidad de Comahue: Neuquén.

Carlino, P. (2009). Enseñar a escribir en la universidad: cómo lo hacen en Estados Unidos y por qué. OEIRevista Iberoamericana de Educación (ISSN: 1681-5653)

Cassany, D. (2011). Construir la escritura. Paidós: Madrid.

Cassany, D. (2012), Reparar la escritura. Didáctica de la corrección de lo escrito. Editorial Graó: Barcelona.

Castillo, S. \& Cabrerizo, J. (2006). Formación del profesorado en educación superior. Desarrollo curricular y evaluación. Vol. II. McGraw-Hill/ Interamericana de España: Madrid.

Cifuentes, R. (2011). Diseño de proyectos de investigación cualitativa. Noveduc: Argentina.

Díaz-Barriga, F., \& Hernández, G. (1999). Estrategias docentes para un aprendizaje significativo. Una interpretación constructivista. 2da edic. McGraw Hill: México.

Educar.ar (S/F). Los Paratextos como guía de lectura. Recuperado el 20 de noviembre 2013 desde: http://www.educ.ar/dinamico/UnidadHtml get_20c3a89c-7a0b-11e1-83aa-ed15e3c494af// index.html.

Esteban, L., Mantelli, N. \& Sivori M. (2009). Nadie escribe para siempre...repensar la escritura como un proceso perfectible. Pp. 289-296.

Flower, L. \& Hayes, J. (1996) "La teoría de la redacción comoprocesocognitivo". Textosencontextol,Bs.As.: Asociación Internacional de Lectura, 73-107. Título 
original: "A cognitive Process Theory of Writing", Theoretical Models and Processes of Reading, Fourth Edition [1994], Rudell, R.; Rapp Rudell, M. y Singer, H. (eds.)

García Negroni, M. \& Tordesillas, M. (2001). La enunciación en la lengua. Madrid: Gredos.

Graves, D. H. (1996). Didáctica de la escritura. 2da. edic. Esiciones Morata, S.L.: Madrid.

Moreles, J., \& Jiménez, S. (2012). La lista y el Journal Club en actividades de lectura y escritura de estudiantes universitarios que realizan tesis. Memorias de ponencias, III Seminario de Lectura en la Universidad; II Congreso Nacional de Expresiones de cultura escrita en instituciones de educación media superior y superior, y IV Seminario Internacional de cultura escrita y actores sociales. 1 al 3 de agosto 2012. México. Pp. 203-210.

Medina, O. (2013). El proceso de producción escrita de la tesis de grado en Arquitectura: una vía para transformar a los estudiantes de pregrado en escritores académicos. (Proyecto de investigación en el Diplomado de lectura y escritura. CEDILEPUCMM).

Padilla, C., Douglas, S. \& López, E. (2010). Yo expongo. Taller de prácticas de comprensión y producción de textos expositivos. Comunicarte: Argentina.

Padilla, C., Douglas, S. \& López, E. (2011). Yo argumento. Taller de prácticas de comprensión $y$ producción de textos argumentativos. Comunicarte: Argentina.

Sanjurjo, L. \& Vera M. (2003). Aprendizaje significativo $y$ enseñanza en los niveles medio y superior. Homo Sapiens ediciones: Argentina.

Van Diik, T. (1980). Texto y contexto. Semántica y pragmática del discurso. Ediciones Cátedra, S.A.: Madrid

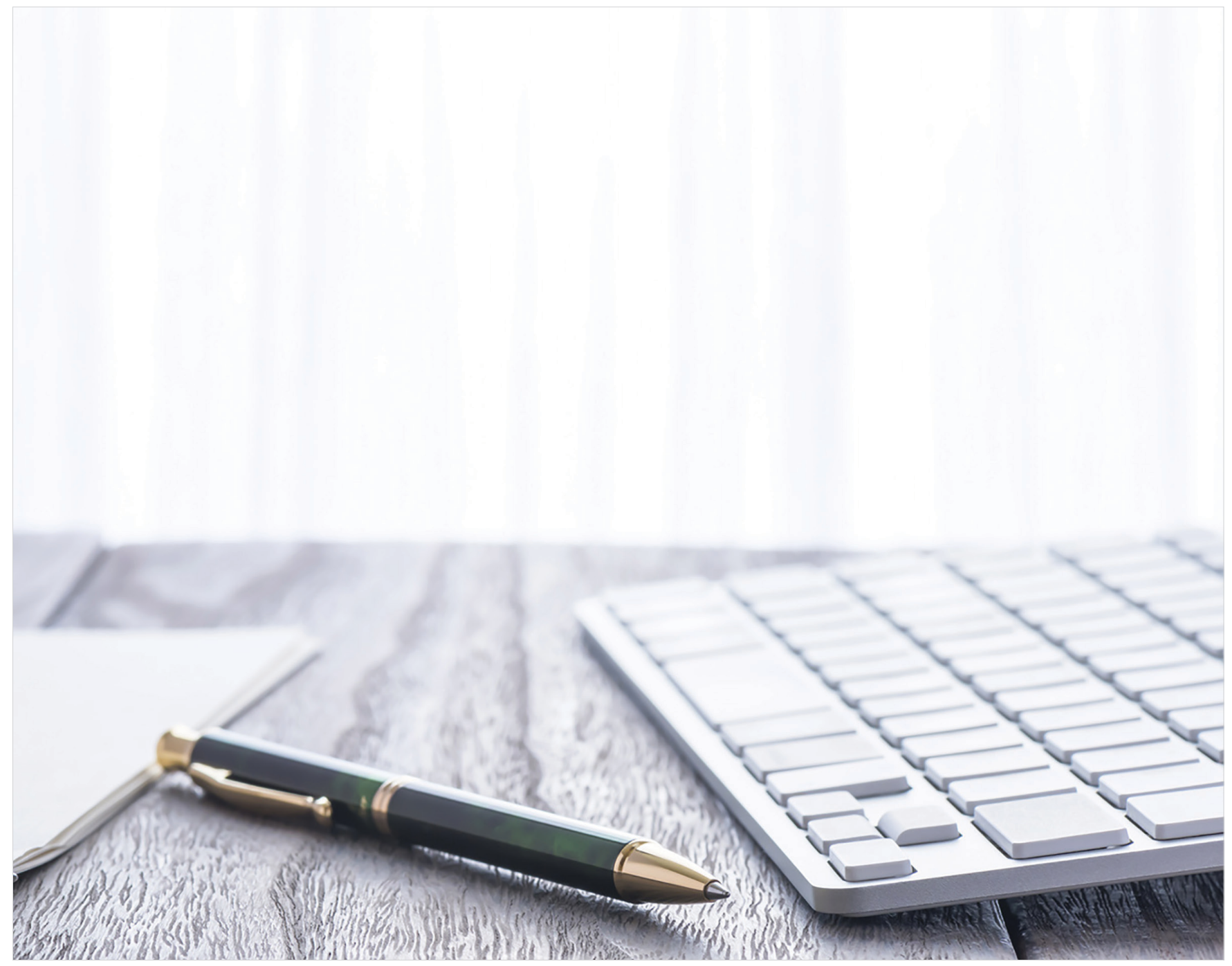

